Pure and Applied Mathematics Quarterly

Volume 6, Number 4

(Special Issue: In honor of

Joseph J. Kohn, Part 2 of 2)

999-1012, 2010

\title{
A Form of Alexandrov-Fenchel Inequality
}

\author{
Pengfei Guan, Xi-Nan Ma, Neil Trudinger and Xiaohua Zhu \\ Dedicated to Professor J.J.Kohn on the occasion of his 75th birthday
}

\begin{abstract}
We give a proof of the Alexandrov-Fenchel type inequality for
\end{abstract} $k$-convex functions on $\mathbb{S}^{n}$.

\section{INTRODUCTION}

There are many important integral formulae and integral inequalities for convex bodies (see [4], [18]). The Brunn-Minkowski inequality and Alexandrov-Fenchel inequality are among the most important integral inequalities in the theory of convex bodies, and the Minkowski type integral formulae and more general formulae of Chern are very useful in the global geometry of convex hypersurfaces. Most of these formulae and inequalities can be stated in the integral forms on the unit sphere $S^{n}$ with the convexity assumption. It seems of interest to establish similar results without the convexity assumption. For a convex body, the polar of the body is also convex. The support function of the convex body corresponds to the gauge function of its polar body. In other words, the geometry of a convex body can be reflected from its polar dual. With this relation, we will introduce a class of domains in $\mathbb{R}^{n+1}$ called $k^{*}$-convex (see Definition 4.2 ) as a natural generalization of convex bodies. We will derive a form of the Alexandrov-Fenchel

Received November 27, 2007.

Research of the first author was supported in part by an NSERC Discovery Grant. Research of the second author was supported by FokYingTung Eduction Foundation, the grants from Ministry of Eduction of China and NSFC No.10371041. Research of the third author was supported by the Australian Research Council. Research of the fourth author was partially supported by NSFC No. 10425102 in China. 
inequality for this class of domains. The concept of $k^{*}$-convexity is related to a Hessian equation on $S^{n}$ by the distance function. It will also be shown that the assumption of convexity is not necessary for some of the integral formulae. We will also prove a uniqueness theorem for the Hessian equation, which generalizes the classical Alexandrov-Fenchel-Jessen theorem.

In most cases, our proofs are not so different from those known in the convex case with two exceptions. First, we work directly on the functions and related vector-valued forms on $S^{n}$ without convexity assumptions. Secondly, we make use of hyperbolic polynomial theory instead of Alexandrov's mixed discriminant inequality, which enables us to replace the notion of convexity by the more general notions. Our arguments are drawn mainly from three important papers: Garding [9], Chern [7] and Cheng-Yau [6]. In fact, the hyperbolic polynomial theory was already used by Chern in [7] in the proof the uniqueness theorem and in Hörmander [14] in the proof of Alexandrov-Fenchel inequality for convex bodies. It should be noted that the hyperbolicity of the elementary symmetric functions was used in the development of fully nonlinear equations in the pioneering work of Caffarelli-Nirenberg-Spruck [5]. So, it is not a coincidence that this theory is used here in a crucial way.

\section{Integral Formulas For the FUnCtions ON $S^{n}$}

Let $e_{1}, \ldots, e_{n}$ is an orthonormal frame on $S^{n}$, let $\omega_{1}, \ldots, \omega_{n}$ be the corresponding dual 1-forms. For each function $u \in C^{2}\left(S^{n}\right)$, let $u_{i}$ be the covariant derivative of $u$ with respect to $e_{i}$. We define a vector valued function

$$
Z=\sum_{i=1}^{n} u_{i} e_{i}+u e_{n+1}
$$

where $e_{n+1}$ is the position vector on $S^{n}$, that is, the outer normal vector field of $S^{n}$. We note that $Z$ is globally defined on $S^{n}$. We write the Hessian matrix of $u$ with respect to the frame as

$$
W=\left\{u_{i j}+u \delta_{i j}\right\} .
$$


By calculation we have,

$$
\begin{aligned}
u= & Z \cdot e_{n+1}, \\
d Z= & \sum_{i=1}^{n}\left(d u_{i} e_{i}+u_{i} d e_{i}\right)+d u e_{n+1}+u d e_{n+1} \\
= & \sum_{i=1}^{n}\left(\sum_{j=1}^{n} u_{i j} \omega^{j}-\sum_{j=1}^{n} u_{j} \omega_{j}^{i}\right) e_{i}+\sum_{i=1}^{n}\left(\sum_{\alpha=1}^{n+1} u_{i} \omega_{i}^{\alpha} e_{\alpha}\right) \\
& +\sum_{i=1}^{n}\left(u_{i} \omega^{i}\right) e_{n+1}+u \sum_{i=1}^{n} \omega^{i} e_{i} \\
= & \sum_{j=1}^{n}\left(\sum_{i=1}^{n}\left(u_{i j}+\delta_{i j} u\right) e_{i}\right) \omega^{j} .
\end{aligned}
$$

For $u^{1}, \ldots, u^{n+1} \in C^{2}\left(S^{n}\right)$, we define $\forall l=1, \ldots, n+1$,

$$
Z^{l}=\sum_{i=1}^{n} u_{i}^{l} e_{i}+u^{l} e_{n+1}
$$

and

$$
W^{l}=\left\{u_{i j}^{l}+u^{l} \delta_{i j}\right\}
$$

Set,

$$
\Omega\left(u^{1}, \ldots, u^{n+1}\right)=\left(Z^{1}, d Z^{2}, d Z^{3}, \ldots, d Z^{n+1}\right),
$$

and

$$
V\left(u^{1}, u^{2} \ldots, u^{n+1}\right)=\int_{S^{n}} \Omega\left(u^{1}, \ldots, u^{n+1}\right)
$$

We note that

$$
\Omega\left(u^{1}, \ldots, u^{n+1}\right)=u^{1} S_{n}\left(W^{2}, \ldots, W^{n+1}\right) d s,
$$

where $S_{n}\left(W^{2}, \ldots, W^{n+1}\right)$ is the mixed determinant and $d s$ is the standard area form on $S^{n}$. In particular, $\forall 1 \leq k \leq n$, if we set $u^{k+2}=\ldots=u^{n+1}=1$, we obtain

$$
\Omega\left(u^{1}, \ldots, u^{n+1}\right)=u^{1} S_{k}\left(W^{2}, \ldots, W^{k+1}\right) d s,
$$

where $S_{k}\left(W^{2}, \ldots, W^{k+1}\right)$ is the complete polarization of the symmetric function $S_{k}$ on symmetric matrices.

Lemma 2.1. $V$ is a symmetric multilinear form on $\left(C^{2}\left(S^{n}\right)\right)^{n+1}$. 
Proof. The multilinearility follows directly from the definition. Also, by the definition, for any permutation $\sigma$ of $\{2, \ldots, n+1\}$,

$$
\Omega\left(u^{1}, u^{2} \ldots, u^{n+1}\right)=\Omega\left(u^{1}, u^{\sigma(2)} \ldots, u^{\sigma(n+1)}\right),
$$

so $V\left(u^{1}, u^{2} \ldots, u^{n+1}\right)=V\left(u^{1}, u^{\sigma(2)} \ldots, u^{\sigma(n+1)}\right)$. To see $V$ is a symmetric form, we only need to show

$$
V\left(u^{1}, u^{2}, u^{3} \ldots, u^{n+1}\right)=V\left(u^{2}, u^{1}, u^{3} \ldots, u^{n+1}\right) .
$$

We first assume $u^{i} \in C^{3}\left(S^{n}\right), \forall i$. Letting,

$$
\omega\left(u^{1}, \ldots, u^{n+1}\right)=\left(Z^{1}, Z^{2}, d Z^{3}, \ldots, d Z^{n+1}\right),
$$

we have

$$
d \omega\left(u^{1}, \ldots, u^{n+1}\right)=-\Omega\left(u^{2}, u^{1}, u^{3} \ldots, u^{n+1}\right)+\Omega\left(u^{1}, u^{2}, u^{3} \ldots, u^{n+1}\right),
$$

whence, (2.5) follows from Stokes theorem. The identity (2.5) is valid for $C^{2}$ function by approximation.

Remark: If $u^{1}, \ldots, u^{n+1}$ are the support functions of convex bodies $K_{1}, \ldots, K_{n+1}$ respectively, then $V\left(u^{1}, u^{2} \ldots, u^{n+1}\right)$ is the Minkowski mixed volume $V\left(K_{1}, \ldots, K_{n+1}\right)$.

The following is a direct corollary of the Lemma 2.1. If $u$ is a support function of a convex body, it is a well known Minkowski type integral formula.

Corollary 2.2. For any function $u \in C^{2}\left(S^{n}\right), W=\left\{u_{i j}+\delta_{i j} u\right\}$. For any $1 \leq k<n$, we have the Minkowski type integral formulae.

$$
\int_{S^{n}} u S_{k}(W) d s=\int_{S^{n}} S_{k+1}(W) d s,
$$

where ds is the standard area element on $S^{n}$.

For any $n \times n$ symmetric matrices $W_{1}, \ldots, W_{k}$, let $S_{k}\left(W_{1}, \ldots, W_{k}\right)$ be the complete polarization of $S_{k}$. Let $u$ and $\tilde{u}$ be two $C^{2}$ functions on $S^{n}$ and let $W$ and $\tilde{W}$ be the corresponding Hessian matrices of $u$ and $\tilde{u}$ respectively. Following Chern's notations in [7], we let $P_{r s}=S_{r+s}(W, \ldots, W, \tilde{W}, \ldots \tilde{W})$ where $W$ appears $r$ times and $\tilde{W}$ appears $s$ times. So, $P_{r s}$ is a polynomial in $W_{i j}, \tilde{W}_{i j}$, homogeneous of degrees $r$ and $s$ respectively. The following dual generalization of Chern's formulae is another corollary of Lemma 2.1 . 
Corollary 2.3. Suppose $u$ and $\tilde{u}$ are two $C^{2}$ functions on $S^{n}$. Then the following identities hold,

$$
\begin{aligned}
& \int_{S^{n}}\left[u P_{0 k}-\tilde{u} P_{1, k-1}\right] d x=0, \\
& \int_{S^{n}}\left[u P_{k-1,1}-\tilde{u} P_{k 0}\right] d x=0,
\end{aligned}
$$

and

$$
\begin{aligned}
& 2 \int_{S^{n}} u\left(P_{0 k}-P_{k-1,1}\right) d x \\
& =\int_{S^{n}}\left\{\tilde{u}\left(P_{1, k-1}-P_{k 0}\right)-u\left(P_{k-1,1}-P_{0 k}\right)\right\} d x=0 .
\end{aligned}
$$

\section{3. $k$-CONVEX FUNCTIONS ON $S^{n}$}

Now we consider functions satisfying the following equation,

$$
S_{k}(W)=\varphi \quad \text { on } \quad S^{n} .
$$

Definition 3.1. For $1 \leq k \leq n$, let $\Gamma_{k}$ be the convex cone in $\mathbb{R}^{n}$ determined by

$$
\Gamma_{k}=\left\{\lambda \in \mathbb{R}^{n}: \quad S_{1}(\lambda)>0, \ldots, S_{k}(\lambda)>0\right\} .
$$

Suppose $u \in C^{2}\left(S^{n}\right)$, we say $u$ is $k$-convex, if $W(x)=\left\{u_{i j}(x)+u(x) \delta_{i j}\right\}$ is in $\Gamma_{k}$ for each $x \in S^{n}$. $u$ is convex on $S^{n}$ if $W$ is semi-positive definite on $S^{n}$. Furthermore, $u$ is called an admissible solution of (3.1), if $u$ is $k$-convex and satisfies (3.1).

The next is a uniqueness theorem which generalizes the Alexandrov-FenchelJessen theorem ([2], [8] and [7]) to the $k$-convex case.

Theorem 3.2. Suppose $u$ and $\tilde{u}$ are two nonnegative $C^{2} k$-convex functions on $S^{n}$ satisfying (3.1). If $S_{k}(W)=S_{k}(\tilde{W})$, then $u-\tilde{u} \in \operatorname{Span}\left\{x_{1}, \ldots, x_{n+1}\right\}$ on $S^{n}$.

Proof of Theorem 3.2. We follow the same lines as in [7]. Since $u$ is nonnegative and $S_{k}(W)$ is positive, we conclude that $u$ is positive almost everywhere on $S^{n}$. By the hyperbolicity of $S_{k}, \forall W^{i} \in \Gamma_{k}, i=1, \ldots, k$,

$$
S_{k}\left(W^{1}, \ldots, W^{k}\right) \geq S_{k}\left(W^{1}\right) \cdots S_{k}\left(W^{k}\right),
$$


with the equality holds if and only if the $k$ matrices are pairwise proportional.

Suppose $S_{k}(W)=S_{k}(\tilde{W})$ on $S^{n}$, where $W=\left\{u_{i j}+\delta_{i j} u\right\}$ and $\tilde{W}=\left\{\tilde{u}_{i j}+\delta_{i j} \tilde{u}\right\}$ The left hand side of the integral formula (2.9) in Corollary 2.3 is non-positive. The same is therefore true of the right hand side of (2.9). The latter is antisymmetric on the two function $u$ and $\tilde{u}$, and hence must be zero. It follows that $P_{k-1,1}=P_{0 k}$ by (3.2). Again, the equality gives that $W$ and $\tilde{W}$ are proportional. Since $S_{k}(W)=S_{k}(\tilde{W})$, we conclude that $W=\tilde{W}$ at each point of $S^{n}$, that is, $u-\tilde{u} \in \operatorname{Span}\left\{x_{1}, \ldots, x_{n+1}\right\}$.

The following is an infinitesimal version of Theorem 3.2, which we will use in our proof of the generalized Alexandrov-Fenchel inequality.

Proposition 3.3. For any $C^{2}$ function $u$, let $L_{u}$ be the linearized operator of the Hessian operator $S_{k}\left(\left\{u_{i j}+\delta_{i j} u\right\}\right)$. Then $L_{u}$ is self-adjoint. If in addition, $u$ is nonnegative admissible solution of (3.1), the kernel of $L_{u}$ is $\operatorname{Span}\left\{x_{1}, \ldots, x_{n+1}\right\}$.

When $u$ is a support function of some convex body, the above proposition is well-known (e.g., see [16]). The proposition is a special case of the following result.

Proposition 3.4. $\forall u^{2}, \ldots, u^{k} \in C^{2}\left(S^{n}\right)$ fixed, define

$$
L(v)=\Omega\left(1, v, u^{2} \ldots, u^{k}, 1, \ldots, 1\right),
$$

then, $L$ is self-adjoint. If in addition, $u^{2}, \ldots, u^{k}$ are $k$-convex, and at least one of them is nonnegative, the kernel of $L$ is $\operatorname{Span}\left\{x_{1}, \ldots, x_{n+1}\right\}$.

Proof of Proposition 3.4. First if $u \in C^{3}$, the linearized operator $L_{u}$ of $S_{k}$ is self-adjoint (see, e.g., [17]). Let $t_{2}, \ldots, t_{k}$ be real numbers, let $u_{t}=\sum_{l=2}^{k} t_{l} u^{l}$, the operator $L$ in (3.3) is the coefficient of the linearized operator $L_{u_{t}}$ of $t_{2} \cdots t_{k}$. Since $L_{u_{t}}$ is self-adjoint for all $t=\left(t_{2}, \ldots, t_{k}\right)$, we conclude that $L$ is self-adjoint,if $u^{l} \in C^{3}, \forall 2 \leq l \leq k$. By approximation, the same conclusion is true for $C^{2}$ functions.

To compute the kernel, we may assume $u^{2}$ is nonnegative. Since $u^{2}$ is $k$-convex, it is positive almost everywhere. Suppose $v$ is in kernel of $L$, i.e.,

$$
L(v)=0 .
$$


Simple calculation shows that

$$
\Omega\left(1, v, v, u^{3}, \ldots, u^{k}, 1, \ldots, 1\right)=S_{k}\left(A, A, W^{3}, \ldots, W^{k}\right) d s,
$$

where $A=\left\{v_{i j}+\delta_{i j} v\right\}$ and $W^{l}=\left\{u_{i j}^{l}+\delta_{i j} u^{l}\right\}$.

We claim that, if (3.4) holds, then

$$
S_{k}\left(A, A, W^{3}, \ldots, W^{k}\right) \leq 0,
$$

with equality if and only if $A=0$, i.e., $v \in \operatorname{Span}\left\{x_{1}, \ldots, x_{n+1}\right\}$.

We note that,

$$
\begin{aligned}
0 & =\int_{S^{n}} v L(v)=\int_{S^{n}} \Omega\left(v, v, u^{2}, u^{3}, \ldots, u^{k}, 1, \ldots, 1\right) \\
& =V\left(v, v, u^{2}, u^{3}, \ldots, u^{k}, 1, \ldots, 1\right)=V\left(u^{2}, v, v, u^{3}, \ldots, u^{k}, 1, \ldots, 1\right) \\
& =\int_{S^{n}} u^{2} \Omega\left(1, v, v, u^{3}, \ldots, u^{k}, 1, \ldots, 1\right) \\
& =\int_{S^{n}} u^{2} S_{k}\left(A, A, W^{3}, \ldots, W^{k}\right) d s .
\end{aligned}
$$

If the claim is true, we will conclude that $v$ is in $\operatorname{Span}\left\{x_{1}, \ldots, x_{n+1}\right\}$ since $u^{2}$ is positive almost everywhere.

To prove the claim, we make use of the result of Garding [9] result on hyperbolicity of $S_{k}$ in the cone $\Gamma_{k}$ (see also [14]). Since $u^{l}$ is $k$-convex, $W^{l} \in \Gamma_{k}, \forall 2 \leq l \leq k$. For $W^{3}, \ldots, W^{k}$ fixed, the polarization $S_{k}\left(B, B, W^{3}, \ldots, W^{k}\right)$ is also hyperbolic and complete for $B \in \Gamma_{k}$. Let $W_{t}=W^{2}+t A$, we have

$$
\begin{aligned}
& S_{k}\left(W_{t}, W_{t}, W^{3}, \ldots W^{k}\right)=S_{k}\left(W^{2}, W^{2}, W^{3}, \ldots, W^{k}\right) \\
& +2 t S_{k}\left(A, W^{2}, W^{3}, \ldots, W^{k}\right)+t^{2} S_{k}\left(A, A, W^{3}, \ldots, W^{k}\right) .
\end{aligned}
$$

Since

$$
S_{k}\left(W^{2}, W^{2}, W^{3} . ., W^{k}\right)>0,
$$

and

$$
S_{k}\left(A, W^{2}, \ldots, W^{k}\right)=0 .
$$

By the hyperbolicity, $S_{k}\left(W_{t}, W_{t}, W^{3}, \ldots, W^{k}\right)$ has only real roots in $t$ variable, so (3.5) must be true. If in addition, $S_{k}(A, A, W, \ldots, W)=0$, we would have

$$
S_{k}\left(W_{t}, W_{t}, W, \ldots, W\right)=S_{k}(W, \ldots, W),
$$

for all $t \in \mathbb{R}$. By the completeness, $A=0$. The claim is proved. 


\section{4. $k^{*}$-CONVEX BODIES AND AleXANDROV-Fenchel INEQUALity}

For any $n \geq k \geq 1$ fixed, set $u^{k+2}=\ldots=u^{n+1}=1$ we define $\forall u^{1}, \ldots, u^{k+1} \in$ $C^{2}\left(S^{n}\right)$

$$
V_{k+1}\left(u^{1}, u^{2}, \ldots, u^{k+1}\right)=V\left(u^{1}, u^{2}, \ldots, u^{n+1}\right) .
$$

Now we state a form of Alexandrov-Fenchel inequality for positive $k$-convex functions.

Theorem 4.1. If $u^{1}, \ldots, u^{k}$ are $k$-convex, and $u_{1}$ positive, and at least one of $u^{l}$ is nonnegative on $S^{n}$ (for $2 \leq l \leq k$ ), then $\forall v \in C^{2}\left(S^{n}\right)$,

$$
V_{k+1}^{2}\left(v, u^{1}, \ldots, u^{k}\right) \geq V_{k+1}\left(u^{1}, u^{1}, u^{2}, \ldots, u^{k}\right) V_{k+1}\left(v, v, u^{2}, \ldots, u^{k}\right),
$$

the equality holds if and only if $v=a u^{1}+\sum_{i=1}^{n+1} a_{i} x_{i}$ for some constants $a, a_{1}, \ldots, a_{n+1}$.

Our proof of the theorem follows the similar arguments of Alexandrov's second proof of Alexandrov-Fenchel inequality in [2] (see also [14]), which in turn is adapted from Hilbert's proof of the Brunn-Minkowski inequality when $n=3$. Instead of using Alexandrov's inequality for mixed discriminants in his original proof, we will make use of the hyperbolicity of the elementary symmetric functions as in [14]. This replacement enables us to drop the convexity assumption. We sketch here some key steps.

\section{Proof.}

\section{Statement: If}

$$
V_{k+1}\left(v, u^{1}, u^{2}, \ldots, u^{k}\right)=0, \quad \text { for some } \quad v \in C^{2}\left(S^{n}\right),
$$

then

$$
V_{k+1}\left(v, v, u^{2}, \ldots, u^{k}\right) \leq 0,
$$

with equality if and only if $v=\sum_{i=1}^{n+1} a_{i} x_{i}$.

The theorem follows directly from above statement. The proof of the Statement will be reduced to an eigenvalue problem for certain elliptic differential operators.

First, for $u^{2}, \ldots, u^{k} \in \Gamma_{k}$ fixed, we set

$$
L(v)=\Omega\left(1, v, u^{2} \ldots, u^{k}, 1, \ldots, 1\right) .
$$


By Garding [9] $L(v)>0$ if $v$ is $k$-convex. We claim that $L$ is an elliptic differential operator with negative principal symbol. This can be done following the same line as in [14]. The principal symbol of $L$ at the co-tangent vector $\theta=\left(\theta_{1}, \ldots, \theta_{n}\right)$ is obtained when $A$ is replaced by $-\theta \otimes \theta$ in

$$
S_{k}\left(A, W^{2}, \ldots, W^{k}\right) .
$$

So it is equal to

$$
-S_{k}\left(\theta \otimes \theta, W^{2}, \ldots, W^{k}\right) .
$$

Since $S_{k}$ is hyperbolic with respect to the positive cone $\Gamma_{k}$, and $\theta \otimes \theta$ is semipositive definite and is not a 0 matrix if $\theta$ not 0 . By the complete hyperbolicity,

$$
-S_{k}\left(\theta \otimes \theta, W^{2}, \ldots, W^{k}\right)<0 .
$$

We now use continuity method to finish the job. For $0 \leq t \leq 1$, let $u_{t}^{i}=$ $(1-t)+t u^{i}$, and set

$$
\rho_{t}=\frac{\Omega\left(1, u_{t}^{1}, u_{t}^{2} \ldots, u_{t}^{k}, 1, \ldots, 1\right)}{u_{t}^{1}},
$$

We examine the eigenvalue problem:

$$
L_{t}(v)=\lambda \rho_{t} v .
$$

If for we set $Q_{t}(u, v)=\int_{S^{n}} u L_{t}(v)$, the eigenvalue problem (4.5) is corresponding to the quadratic form $Q_{t}$ with respect to the inner-product $\left\langle u, v>_{\rho_{t}}=\int_{S^{n}} u v \rho_{t}\right.$.

We want to show Claim: $\lambda=1$ is the only positive eigenvalue of multiplicity 1 with eigenfunction $u_{t}^{1}$, and $\lambda=0$ is the eigenvalue of multiplicity $n+1$ with the eigenspace $\operatorname{Span}\left\{x_{1}, \ldots, x_{n+1}\right\}$ for the eigenvalue problem of (4.5).

We note that $u_{t}^{1}$ is an eigenfunction corresponding to the eigenvalue $\lambda=1$. If the Claim is true, (4.3) implies that $v$ is orthogonal to the eigenspace corresponding to $\lambda=1$ with respect to the inner product $<., .>_{\rho_{1}}$. Then the Statement follows from the standard spectral theory of self-adjoint elliptic operators.

We now prove the Claim. When $t=0$, the problem can be reduced to the following simple form by straightforward calculations:

$$
\Delta v+n v=n \lambda v .
$$

The eigenvectors of $\Delta$ are the spherical harmonics of degree $\nu=0,1, \ldots$, with the corresponding eigenvalues $-\nu(\nu+n-1) . \nu=0$ corresponds to $\lambda=1$ and $\nu=1$ corresponds to $\lambda=0$ in the eigenvalue problem (4.5) respectively in this 
special case. And $\lambda<0$ when $\nu>1$. It is well known that spherical harmonics of degree 0 are constants, and spherical harmonics of degree 1 are linear functions, i.e., $\operatorname{Span}\left\{x_{1}, \ldots, x_{n+1}\right\}$. Therefore, the Claim is true for $t=0$. For arbitrary $t$, since 1 is an eigenvalue of the problem (4.5) with eigenfunction $u_{t}^{1}$, by the theory of elliptic equations, we only need to prove that 0 is the eigenvalue of multiplicity $n+1$. It's obvious that $x_{1}, \ldots, x_{n+1}$ are the eigenfunctions of $L$ corresponding to the eigenvalue 0. The theorem now follows from Proposition 3.4.

Now, we consider a class of domains which will be named $k^{*}$-convex. They can be viewed as a generalization of convex bodies via polar dual. Let $D$ be a star-shaped bounded domain in $\mathbb{R}^{n+1}$ with $C^{2}$ boundary. The distance function of $D$ is defined as,

$$
u(x)=\min \{\lambda \mid x \in \lambda D\}, \quad \forall x \in S^{n} .
$$

When $D$ is convex, the distance function is also called the gauge function of $D$.

Definition 4.2. Let $D$ be a star-shaped bounded domain in $\mathbb{R}^{n+1}$ with $C^{2}$ boundary. We say $D$ is $k^{*}$-convex if its distance function $u$ is $k$-convex on $S^{n}$. We say $D$ is polar centrized if its distance function $u$ satisfies

$$
\int_{S^{n}} x_{j} u(x) d s=0, \quad \forall j=1,2, \ldots, n+1 .
$$

If $D_{1}, \ldots, D_{k+1}$ are $k^{*}$-convex bodies, let $u_{1}, \ldots, u_{k+1}$ are the corresponding distance functions, and $W_{1}, \ldots, W_{k+1}$ be the corresponding Hessians of the gauge functions. For $0 \leq l \leq k$, we define mixed polar surface area functions

$$
S_{l}\left(D_{1}, \ldots, D_{l}, x\right)=S_{l}\left(W_{1}, \ldots, W_{l}\right) .
$$

We call $S_{l}(D, x)=S_{l}(W, \ldots, W)$ the $l$ th polar surface are function of $D$. We also define a mixed polar volume,

$$
V_{k+1}^{*}\left(D_{1}, \ldots, D_{k+1}\right)=\frac{1}{V_{k+1}\left(u_{1}, \ldots, u_{k+1}\right)}
$$

where $V_{k+1}\left(u_{1}, \ldots, u_{k+1}\right)$ defined as in (4.1). We also write, $\forall 0 \leq l \leq k+1$, $V_{l}^{*}(D)=V_{k+1}^{*}(D, \ldots, D, B, \ldots, B)$, where $B$ is the unit ball centered at the origin in $\mathbb{R}^{n+1}, D$ appears $l$ times, and $B$ appears $k+1-l$ times in the formula. 
We note that if $D$ is convex, $D$ is polar centerized if and only if the Steiner point of the polar of $D$ is the origin. If $D$ is convex, $V_{l}^{*}(D)$ in Definition 4.2 is the reciprocal of the $l$ th quermassintegral of the polar of $D$. These geometric quantities of $D$ and its polar $D^{*}$ in this case are related by some important inequalities, like the Blaschke-Santalo inequality.

As an application, we have the following consequences of Theorem 3.2 and Theorem 4.1.

Theorem 4.3. Suppose $D_{1}, D_{2}$ are two $k^{*}$-convex domains in $\mathbb{R}^{n+1}$. If the $k$ th polar surface area functions of $D_{1}$ and $D_{2}$ are the same, i.e.,

$$
S_{l}\left(D_{1}, x\right)=S_{l}\left(D_{2}, x\right), \quad \forall x \in S^{n},
$$

then, the distance functions of $D_{1}, D_{2}$ are equal up to a linear function. In particular, if both $D_{1}$ and $D_{2}$ are polar centrized, then $D_{1}=D_{2}$.

Theorem 4.4. Suppose $D_{1}, \ldots, D_{k+1}$ are $k^{*}$-convex domains in $\mathbb{R}^{n+1}$, then we have the following Alexandrov-Fenchel inequality for the mixed polar volumes:

$$
\left(V_{k+1}^{*}\left(D_{1}, \ldots, D_{k+1}\right)\right)^{2} \leq V_{k+1}^{*}\left(D_{1}, D_{1}, D_{3} \ldots, D_{k+1}\right) V_{k+1}^{*}\left(D_{2}, D_{2}, D_{3} \ldots, D_{k+1}\right),
$$

with the equality if and only if the distance functions of $D_{1}$ and $D_{2}$ are equal up to a linear function. In particular, if both $D_{1}, D_{2}$ are polar centerized, then $D_{1}=\lambda D_{2}$ for some $\lambda>0$.

The above theorem shows that the reciprocal of the mixed polar volume is log-concave. Therefore, one may deduce a sequence of inequalities for $k^{*}$-convex domains from Theorem 4.1 as in the convex case (see section 20 in [4], section 6.4 in [18] and appendix in [14]). In particular, one can obtain the corresponding Brunn-Minkowski inequality and quermassintegral inequalities for $V^{*}$.

Corollary 4.5. Suppose $D_{1}, D_{2}$ are $k^{*}$-convex, then for $0 \leq t \leq 1$,

$$
V_{k+1}^{*}\left((1-t) D_{1}+t D_{2}\right)^{\frac{-1}{k+1}} \geq(1-t) V_{k+1}^{*}\left(D_{1}\right)^{\frac{-1}{k+1}}+t V_{k+1}^{*}\left(D_{2}\right)^{\frac{-1}{k+1}},
$$

if $D_{1}, D_{2}$ are polar centralized, the equality for some $0<t<1$ holds if and only if $D_{1}=\lambda D_{2}$ for some $\lambda>0$. If $D$ is $k^{*}$-convex, then for $0 \leq i<j<l \leq k+1$,

$$
\left(V_{j}^{*}(D)\right)^{l-i} \leq\left(V_{i}^{*}(D)\right)^{l-j}\left(V_{l}^{*}(D)\right)^{j-i} .
$$


If $D$ is polar centralized, the equality holds if and only if $D$ is a ball centered at the origin. In particular, if we let $\sigma_{n}$ be the volume of the unit ball $B$ in $\mathbb{R}^{n+1}$,

$$
\sigma_{n}^{i-j}\left(V_{j}^{*}(D)\right)^{k-i} \leq\left(V_{i}^{*}(D)\right)^{k-j}
$$

if $D$ is polar centralized, the equality holds if and only if $D$ is a ball centered at the origin.

We discuss some problems arising from the subject we treated.

(1) The first one is prescribing $k^{*}$ surface area function problem. The equivalent analytical problem is to find a positive solution of the equation (3.1). Of course, $\varphi$ has to satisfy $\int_{S^{n}} x \varphi(x)=0$. The existence and regularity of admissible solutions have been established in [13]. The questions is when an admissible solution is positive? We note that if $u$ is a solution of (3.1), $u(x)+l(x)$ is also a solution for any linear function $l(x)$. Therefore, it is required to put some restriction on the solutions. One of that is to require $u$ orthogonal to the span of $x_{1}, \ldots, x_{n+1}$, that corresponds to find a polar centralized $k^{*}$-convex body. The uniqueness of the positive solutions for the problem is a consequence of Theorem 3.2.

(2) Equation (3.1) has another important geometric connection, it is related to the Christoffel-Minkowski problem. In that case, one looks for convex solutions of (3.1). The intermediate Christoffel-Minkowski problem is still unsolved. A sufficient condition was obtained in recent paper [12] (see also [19]).

(3) Equation (3.1) is a model case for general equations of the form:

$$
F\left(\left\{u_{i j}+\delta_{i j} u\right)=\varphi\right.
$$

where $F$ is assumed to be elliptic and concave. When $F$ is a quotient of Hessians, the equation is related to prescribing Weingarten curvatures on outer normals. We refer [3], [7], [10], [13] and [19] for further references.

(4) The paper [20] deals with the k-convex version of the last inequality in Corollary 4.5 , but the proof in general is incomplete. This inequality has been verified for the star shaped case in [11] and for the case $k=1$ under outward minimizing condition in [15]. 


\section{REFERENCES}

[1] A.D. Alexandrov, Zur Theorie der gemischten Volumina von konvexen korpern, II. Neue Ungleichungen zwischen den gemischten Volumina und ihre Anwendungen (in Russian) Mat. Sbornik N.S. 2 (1937), 1205-1238.

[2] A.D. Alexandrov, Zur Theorie der gemischten Volumina von konvexen korpern, III. Die Erweiterung zweeier Lehrsatze Minkowskis uber die konvexen polyeder auf beliebige konvexe Flachen ( in Russian) Mat. Sbornik N.S. 3, (1938), 27-46.

[3] A. D. Alexandrov, Uniqueness theorems for surfaces in the large, I, Vestnik Leningrad. Univ., 11 (1956), 5-17.

[4] Y.D. Burago and V.A. Zalgaller, Geometric Inequalities, Springer, Berlin, 1988.

[5] L. Caffarelli, L. Nirenberg and J. Spruck, The Dirichlet problem for nonlinear second order elliptic equations, III: Functions of the eigenvalues of the Hessian Acta Math. 155, (1985),261 - 301.

[6] S.Y. Cheng and S.T. Yau, On the regularity of the solution of the n-dimensional Minkowski problem Comm. Pure Appl. Math. 29, (1976), 495-516.

[7] S.S. Chern, Integral formulae for hypersurfaces in euclidean space and their applications to uniqueness theorems, J. Math. Mech. 8, (1959), 947-955.

[8] W. Fenchel and B. Jessen, Mengenfunktionen und konvexe korper, Det. Kgl. Danske Videnskab. Selskab, Math.-fys. Medd. 16(3), (1938), 1-31.

[9] L. Garding, An inequality for hyperbolic polynomials, J. Math. Mech. 8, (1959), 957-965.

[10] B. Guan and P. Guan, Convex Hypersurfaces of Prescribed Curvature, Annals of Math. 156, (2002), 655-674.

[11] P. Guan and J. Li, The quermassintegral inequalities for starshaped domains, Advances in Mathematics 221, (2009), 1725-1732.

[12] P. Guan and X. Ma, Christoffel-Minkowski problem I: convexity of solutions of a Hessian equation, Invent. Math. 151, (2003), 553-577.

[13] P. Guan, X.Ma and F.Zhou, Christoffel-Minkowski problem III: Admissible solutions of Hessian equation on $S^{n}$, Comm. on Pure and Applied Math., 59, (2006) 1352-1376.

[14] L. Hörmander, Notions of Convexity, Birkhauser, Boston, (1994).

[15] G. Huisken, private commnication, (2007).

[16] A. V. Pogorelov, The Minkowski multidimensional problem, Wiley, New York, (1978).

[17] R. C. Reilly, On the Hessian of a function and the curvatures of its graph, Michigan Math. J. 20, (1973), 373-383.

[18] R. Schneider, Convex bodies: The Brunn-Minkowski theory, Cambridge University, (1993).

[19] W. Sheng, N. Trudinger and X.J. Wang, Convex hypersurfaces of prescribed Weingarten Curvatures, Comm. Analysis and Geometry 12, (2004), 213-232.

[20] N. Trudinger, Isoperimetric inequalities for quermassintegrals, Ann. Inst. H. Poincarè Anal. Non Linèaire 11, (1994), 411-425. 
Pengfei Guan

Department of Mathematics

McGill University

Montreal, QC. H3A 2K6, Canada

E-mail: guan@math.mcgill.ca

Xi-Nan Ma

Department of Mathematics

East China Normal University

Shanghai, 200062, China

E-mail:xnma@math.ecnu.edu.cn

Neil Trudinger

School of mathematical Sciences

Australia National University

Canberra, Australia

E-mail: neil.trudinger@maths.anu.edu.au

Xiaohua Zhu

School of mathematical Sciences

Beijing University

Beijing, 100871, China

E-mail: xhzhu@math.pku.edu.cn 